\title{
NUEVA FORMULACION DE LA LEY DE GRIMM
}

Jack L. Wilson

En 1822 el érudito alemán Jacob Grimm formalizó las observaciones que antes habían sido hechas por el lingüista danés Rasmus Rask sobre el cambio casi regular de ciertas consonantes en las lenguas germánicas. Rask y Grimm habían notado que las oclusivas aspiradas sonoras bh, dh y gh se convertían respectivamente en las oclusivas sonoras b, d y g; que $\mathrm{b}, \mathrm{d}$ y $\mathrm{g}$ se convertían en las oclusivas $\mathrm{p}, \mathrm{t}$ y $\mathrm{k}$; y que $\mathrm{p}, \mathrm{t}$ y $\mathrm{k}$ se convertían en las fricativas $f, \theta$ y $x$.

A los miembros de la primera serie Grimm dio el nombre de Aspiratae (bh, dh, gh - y $\mathrm{f}, \boldsymbol{\theta}, \mathrm{yx}$, etc.), a los de la segunda serie el nombre de Mediae, y a los de la tercera serie el nombre de Tenues. Grimm produjo un esquema circular "de la siguiente forma:

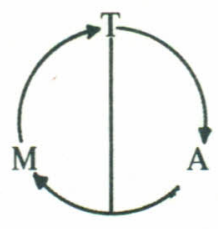

Posteriormente, esta formulación se conoció con el nombre de "Ley de Grimm".

Se suponía que estos cambios no se habían efectuado de manera simultánea, sino en etapas. Dice Thomas Pyles en su libro The origins and development of the English language: "Si bien no podemos estar seguros de la cronología de estos cambios consonánticos, es patente que abarcaron siglos - tal vez un milenio. Cada conjunto de cambios fue completado antes de que el siguiente comenzara; el Primer Cambio de Sonidos no representaba un proceso circular. Es obvio, por ejemplo, que el cambio de b, $\mathrm{d}$ y g del indoeuropeo a $\mathrm{p}, \mathrm{t}$ y $\mathrm{k}$ del germánico tiene que haberse producido mucho después de que $\mathrm{p}, \mathrm{t}$ y $\mathrm{k}$ del indoeuropeo se habían convertido en $\mathrm{f}, \theta$ y $\mathrm{x}$-si no hubiera sido así, la $\mathrm{p}, \mathrm{t}$ y $\mathrm{k}$ germánicas provenientes de $b$, $d$ y $g$ del indoeuropeo habrían continuado cambiando hasta convertirse también en $f, \Theta$ y $\mathrm{x}, \mathrm{y}$ no tendríamos palabras con p.t y k" (pág. 94).

Margaret M. Bryant, en su libro Modern English and its heritage, afirma lo mismo: "Cada grupo original de consonantes cambió solamente una vez. Se cree que el proceso total se produjo en tres etapas. Después del cambio en $\mathrm{p}, \mathrm{t}$ y $\mathrm{k}$, luego fue posible que $\mathrm{b}, \mathrm{d}$ y $\mathrm{g}$ cambiaran sin producirse homófonos causados por la fusión de sonidos que antes separaban las palabras. Después de este segundo cambio, ya era posible que se efectuara el tercero sin causar confusión" (pág. 25). 
Otro tanto afirma Ronald Wardhaugh en su libro Introduction to linguistics: "Una manera de formular el cambio consiste en decir que se produjo una serie de cambios...” (pág. 177).

Por último, John T. Waterman, en su libro Perspectives in linguistics, dice que: "...es obvio que en el cambio del indoeuropeo al germánico cada una de las tres series de consonantes atravesó una etapa o fase del ciclo" (Pág. 28).

De acuerdo con este razonamiento, los cambios formalizados en la ley de Grimm se efectuaron en tres etapas así:

$\begin{array}{cll}\text { Primera etapa } & \text { Latín } & \text { Inglés } \\ \mathrm{p} \Rightarrow \mathrm{f} & \text { pater } & \text { father } \\ \mathrm{t} \Rightarrow \Theta & \text { trēs } & \text { three } \\ \mathrm{k} \Rightarrow \mathrm{x} & \text { cornū } & \text { horn }\end{array}$

Al producirse este primer cambio, ya quedaba libre el espacio ocupado anteriormente por la serie de las oclusivas sordas y éra posible que se efectuara el segundo cambio sin el peligro de que se fusionaran las dos series:

\section{Segunda etapa}

$\mathrm{b} \Rightarrow \mathrm{p}$

$\mathrm{d} \Rightarrow \mathrm{t}$

labium

düo genus

$\operatorname{lip}$

two

$\mathrm{g} \Rightarrow \mathrm{k}$

kin

Ahora se daban las condiciones para que se produjera el tercer cambio, ya que quedaba libre el espacio de las oclusivas sonoras no aspiradas:

$\begin{array}{lll}\text { Tercera etapa } & \\ \text { bh } \Rightarrow \mathrm{b} & \text { frāter } & \text { brother } \\ \mathrm{dh} \Rightarrow \mathrm{d} & \text { foris } & \text { door } \\ \mathrm{gh} \Rightarrow \mathrm{g} & \text { hortus } & \text { garden }\end{array}$

En todo esto es básico el razonamiento de que si los cambios no se hubieran efectuado en tres etapas distintas se habría producido una fusión de los tres conjuntos. No se toma en cuenta ninguna alternativa.

El trabajo que aquí se presenta tiene por objetivo demostrar la falsedad de esta premisa y que todos los cambios bien pudieron haberse realizado en forma simultánea.

Si asignamos rasgos distintivos a los segmentos consonánticos que participaron en los cambios del indoeuropeo al germánico, vemos que en cada caso entran cinco rasgos: vocálico, consonántico, tensión, sonoridad y oclusión. De acuerdo con la teoría de cambios por etapas, primero cambió la polaridad del rasgo oclusivo para convertir a los Tenues en Aspiratae. En la segunda etapa se cambió la polaridad del rasgo de sonoridad en la serie de los Mediae convirtiéndolos en Tenues, y por último cambió la polaridad del rasgo de tensión, convirtiendo a los Aspiratae en Mediae. Esquemáticámente:

$$
\begin{aligned}
& \text { Primera etapa } \\
& \left|\begin{array}{l}
\text {-sonoro } \\
\text {-tenso } \\
\text { toclusivo }
\end{array}\right| \longrightarrow\left|\begin{array}{l}
\text {-sonoro } \\
\text {-tenso } \\
\text {-oclusivo }
\end{array}\right| \\
& \text { Segunda etapa } \\
& \left|\begin{array}{l}
\text {-tenso } \\
\text { toclusivo } \\
\text { +sonoro }
\end{array}\right| \longrightarrow\left|\begin{array}{l}
\text {-tenso } \\
\text { toclusivo } \\
\text {-sonoro }
\end{array}\right|
\end{aligned}
$$




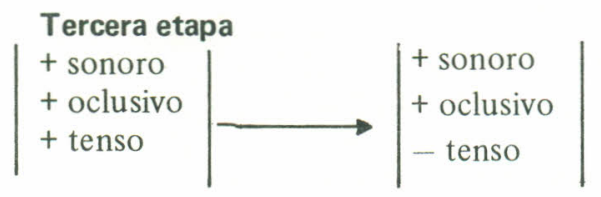

En cada caso vemos que ha habido un cambio de polaridad de un solo rasgo, y en forma aparentemente desordenada.

En vista de que todos los segmentos tienen representaciones de estos rasgos (sea en forma de $+\mathrm{o}-$ en un sistema binario), es posible incluir los rasgos en una matriz que caracterice tanto las consonantes del indoeuropeo como las del germánico, pero en este caso es necesario que los rasgos tengan un ordenamiento jerárquico:

$$
\left|\begin{array}{ll}
- \text { vocálico } \\
+ \text { consonántico } \\
\alpha & \text { oclusivo } \\
\beta & \text { sonoro } \\
\gamma & \text { tenso }
\end{array}\right|
$$

En este esquema las letras griegas representan variables con un valor de + :

Con este procedimiento, es obvio el proceso que siguió la serie de cambios del indoeuropeo al germánico. A los variables en la matriz hay que asignarles condiciones de la siguiente manera:

Si $\gamma=+, \beta=+$

Si $\beta=+, \alpha=+$

Esto significa en forma gráfica que:

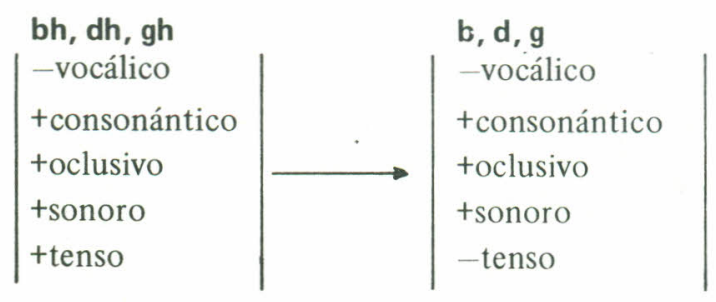

\begin{tabular}{l|l}
$\mathbf{b}, \mathbf{d}, \mathbf{g}$ \\
-vocálico \\
+consonántico \\
+oclusivo \\
+sonoro \\
-tenso
\end{tabular}$|\longrightarrow| \begin{aligned} & \mathbf{p}, \mathbf{t}, \mathbf{k} \\
& \text {-vocálico } \\
& \text { +consonántico } \\
& \text { toclusivo } \\
& \text {-sonoro } \\
& \text {-tenso }\end{aligned} \mid$

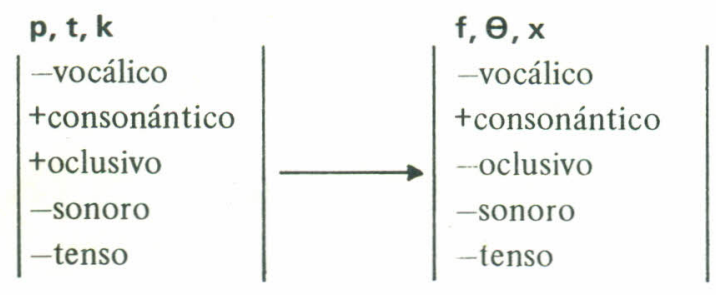


Todo esto significa que los cambios se produjeron en forma simultánea mediante el cambio de polaridad del signo + que se encuentra en la posición más baja en la matriz, y que la siguiente regla representa la formulación correcta del proceso de cambios consonánticos del indoeuropeo al germánico:

$$
\text { R. 1. }+\Rightarrow-1\left|\begin{array}{l}
- \text { vocálico } \\
+X
\end{array}\right| \quad \text { Condición: } X \neq \phi
$$

Si no se hubiera establecido la condición, el rasgo [ + consonántico ] habría cambiado por el de [ - consonántico ] lo cual sería la deșcripción de /h/. Este fue, precisamente, el cambo que se efectuó posteriormente del germánico al inglés al convertirse /x/ en $/ \mathrm{h} /$.

Se debe señalar que la regla que arriba se da, también produciría los cambios que nota Lehmann (pág. 88) de $/ \mathrm{k}^{\mathrm{W}} /$ en $/ \mathrm{x}^{\mathrm{W}} /, \mathrm{gW} /$ en $/ \mathrm{k}^{\mathrm{W}} / \mathrm{y}$ de $/ \mathrm{gW}^{\mathrm{W}} \mathrm{h} /$ en $/ \mathrm{g}^{\mathrm{W}} /$.

En gran parte el latín caracteriza el estado consonántico del indoeuropeo formulado por Grimm y el inglés hace lo mismo para el germánico, en cuanto a estos ejemplos. El único cambio en inglés fue el de $/ \mathrm{x} /$ en $/ \mathrm{h} /$, que se acaba de mencionar. En cuanto al latín los cambios estaban en la serie de los Aspiratae que terminaron en $\mathrm{f}, \mathrm{f}$ y $\mathrm{h}$ provenientes respectivamente de bh, dh y gh. Aquí se ven los cambios más radicales, pues cambió la polaridad de los tres rasgos: oclusión, tensión y sonoridad más dos rasgos distintivos adicionales: la anterioridad y la labialidad. La regla que formaliza el cambio es la siguiente:

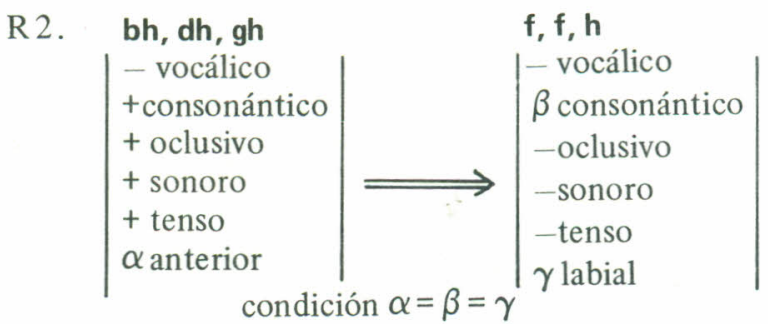

Esta regla establece la concordancia de polaridad entre el rasgo de anterioridad y los rasgos consonántico y labial. Si el valor de $\alpha$ es + (en $\alpha$ anterior), el mismo valor tienen $\beta$ y $\gamma$ en la segunda matriz, y el resultado es la descripción de /f/ (tanto bh y dh como f son segmentos anteriores). Si el valor de $\alpha$ en la primera matriz es -, el mismo valor tienen $\beta$ y $\gamma$ en la segunda, y el resultado es la lista de rasgos distintivos que corresponden a $/ \mathrm{h} /$.

Un proceso similar al que dio origen a la ley de Grimm se realizó en latín en la serie de las oclusivas, produciendo cambios en español, si bien hay una sola diferencia de detalle. La serie que en latín se manifestaba como $-\mathrm{pp}-,-\mathrm{tt}-\mathrm{y}-\mathrm{kk}-$, en posición intervocálica, se manifiesta en español como $-\mathrm{p}-,-\mathrm{t}-\mathrm{y}-\mathrm{k}-$, respectivamente. La serie intervocálica latina $-\mathrm{p}-,-\mathrm{t}-\mathrm{y}-\mathrm{k}-$ dio origen en español a la serie $-\mathrm{b}-,-\mathrm{d}-\mathrm{y}-\mathrm{g}-$, respectivamente. La serie latina $-\mathrm{b}-,-\mathrm{d}-\mathrm{y}-\mathrm{g}-$ se mantuvo en algunos casos, y tuvo una realización $\phi$ en otros. Ejemplos son:

Latín
cŭppa
lǔpu
bībere (en España
probablemente
bibëre)
cantāre hābeo

\section{Español}

copa

lobo

beber

cantaré (habeo $\rightarrow$ aio $\rightarrow$ ai $\rightarrow$ e) 


$\begin{array}{ll}\text { gǔtta } & \text { gota } \\ \text { vita } & \text { vida } \\ \text { jūdex } & \text { juez } \\ \text { siccu } & \text { seco } \\ \text { ficu } & \text { higo } \\ \text { yūgu } & \text { yugo } \\ \text { litigāre } & \text { lidiar }\end{array}$

La serie latina $-\mathrm{p}-,-\mathrm{t}-\mathrm{y}-\mathrm{k}-$ que se convirtió en $-\mathrm{b}-,-\mathrm{d}-\mathrm{y}-\mathrm{g}-$, y la serie $-\mathrm{b}-$, $-d-y-g-$ que se mantuvo, se convirtieron a la vez en una serie fricativa al perder la oclusión en posición intervocálica como rasgo distintivo: $\bullet, \mathrm{t}, \mathrm{y} \frac{8}{\mathrm{~s}}$.

La misma regla R1 que explica los cambios del indoeuropeo al germánico explica estos cambios del latín al español si se cambia el rasgo distintivo 'sonoro' por 'sordo':

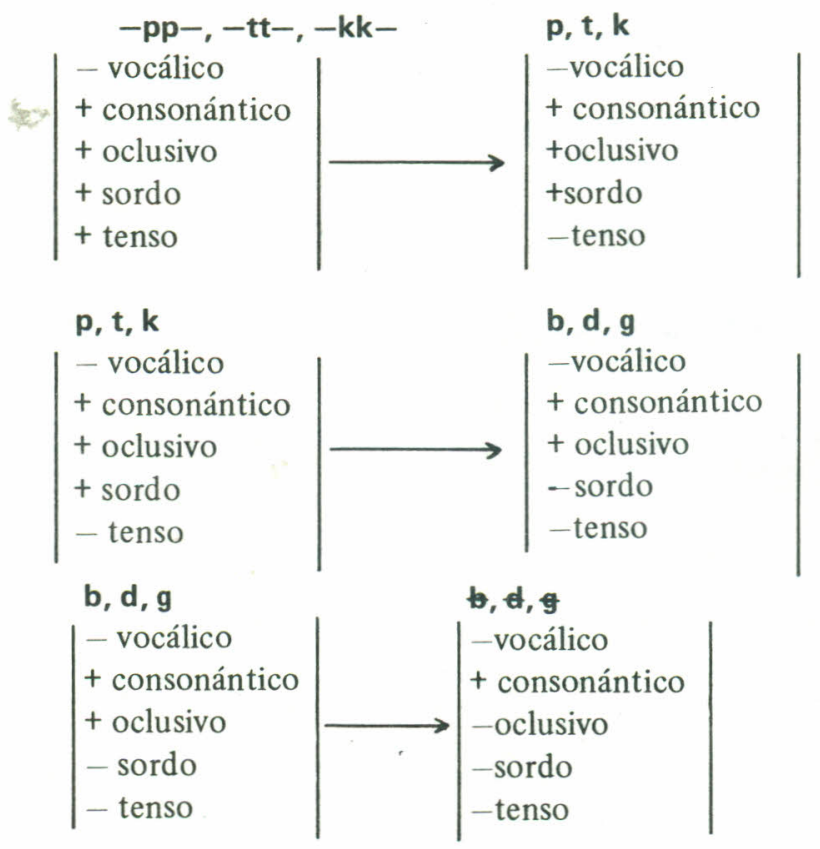

La tendencia de esta última serie ha sido hacia la desaparición, si bien la - b- se ha mantenido más firme. Esta generalización se capta al agregar a la segunda matriz de la última regla la opción siguiente:

$$
+\mathrm{R} \Rightarrow-\mathrm{R}
$$

Esto significa que en forma optativa todos los rasgos (incluyendo el rasgo [ + segmento ]) son -. Con el cambio de detalle del rasgo 'sonoro' por el de 'sordo', los cambios de las series oclusivas del latín al español obedecen a la misma regla que rige para el indoeuropeo al germánico (esta vez sin condición a la regla):

$$
\text { R3. }+\Rightarrow-1\left|\begin{array}{l}
\text {-vocálico } \\
+\mathrm{X}
\end{array}\right|
$$


Ahora, si abandonamos el sistema de rasgos de selección binaria a favor de un sistema de selección $\mathrm{n}$-aria, podemos explicar todos los cambios mencionados (al germánico, al latín, al inglés y al español) como resultado de un relajamiento de tensión muscular que se produjo de manera simultánea en diferentes etapas. La regla general que explica todos estos fenómenos sería:

$$
\text { R4. N tensión } \rightarrow \text { N-1 Tensión / }\left|\begin{array}{l}
- \text { vocálico } \\
+\mathrm{X} \\
-
\end{array}\right|
$$

En conclusión: no hay razón para suponer que los cambios formulados por Grimm se produjeron en tres etapas, como no hay razones para suponer lo mismo de los cambios del latín al español. Parece más razonable que en cada caso los cambios fueran simultáneos al producirse el simple cambio de polaridad de un rasgodistintivo que afectó a los distintos idiomas de manera ligeramente diferente.

\section{REFERENCIAS}

Bryant, Margaret M. 1948. Modern English and its heritage. New York: The MacMillan Company.

Lehmann, Winfred P. 1962. Linguistics: An introduction. New York: Holt, Rinehart, and Winston.

Pyles, Thomas. 1964. The origins and development of the English language. New York: Harcourt, Brace and World.

Wardhaugh, Ronald. 1972. Introduction to linguistics. New York: McGraw Hill Book Company.

Waterman, John T. 1963. Perspectives in linguistics. Chicago: The University of Chicago Press. 